\title{
HEADACHE DISORDERS
}

\section{LANGERHANS CELL HISTIOCYTOSIS AND FRONTAL HEADACHE}

A most recent case record of the Massachusetts General Hospital involved a clinicopathological presentation of a 15-year-old boy admitted with frontal headache, right retro-orbital pain, and a retro-orbital mass that proved to be a Langerhans' cell histiocytosis involving the right sphenoid sinus and orbit. Episodes of pain and headache began 3 weeks before admission and occurred two or three times weekly, lasted one or two hours, were not associated with photophobia or vomiting, and were relieved by ibuprofen. Eight days before admission he had visual impairment in the right eye, and diplopia provoked by looking down and to the left, a trochlear nerve and superior oblique muscle lesion. MRI showed an enhancing right retro-orbital mass that extended into the right sphenoid, posterior ethmoid and right cavernous sinuses, and involved the internal carotid artery. CT revealed a destructive lesion in the right sphenoid bone and optic canal. Visual acuity was $20 / 100$ in the right eye, the optic disks were normal, and downward gaze was impaired in the right eye. A biopsy of the sphenoid sinus revealed aggregates of histiocytic cells and numerous eosinophils. Immunohistochemistry of histiocytes for CD1a was positive and confirmed a Langerhans' cell histiocytosis, with intracytoplasmic Birbeck granules. Treatment with dexamethasone was immediately beneficial, and following the diagnostic biopsy, stereotactic radiation therapy was used for 6 weeks, with improvement in double vision and control of headaches. Differential diagnoses suggested were neoplasm, possible eosinophilic granuloma, and non Hodgkin's lymphoma, probably T-cell or lymphoblastic lymphoma. (Harris NL et al. Clinicopathological exercise, MGH case record. Langerhans' cell histiocytosis and retro-orbital mass. N Engl I Med February 14, 2002;346:513-520).

COMMENT. Langerhans'-cell histiocytosis may present with headache and neurologic symptoms and signs of a mass lesion involving the sphenoid sinus and orbit, without other systemic manifestations.

\section{MIGRAINE AND AUTONOMIC NERVOUS SYSTEM DYSFUNCTION}

Tests of autonomic nervous system (ANS) function were administered to 80 adult patients with migraine ( 28 with disabling headaches) and 85 matched controls, in a study at the Thomas Jefferson University Hospital, Philadelphia, PA, Johns Hopkins School of Hygiene and Public Health, Albert Einstein College of Medicine, and Montefiore Medical Center, Bronx, NY. During headache-free intervals, resting diastolic, but not systolic, blood pressure was elevated in migraineurs with disabling headaches compared to nondisabled patients and controls. Pulse rate variability during deep breathing, and the Valsalva maneuver ratio, a measure of change in heart rate, were significantly lower in disabled migraineurs of nondisabled and controls. Blood pressure response to psychological stress was not different in the 3 groups. Migraineurs with disabling headaches may be susceptible to impaired autonomic nervous responses. The link between migraine and abnormal autonomic function is unclear. The dysfunction of the ANS may increase the tendency to headaches by lowering the threshold to migraine triggers. (Shechter A, Stewart WF, Silberstein SD, Lipton RB. Migraine and autonomic nervous system function. A population-based, case-control study. Neurology February (1 of 2) 2002;58:422-427). (Reprints: Aaron Shechter, Jefferson Headache Center, Department of Neurology, Thomas Jefferson University Hospital, $111 \mathrm{~S} 11$ th 